\title{
Creation and Annihilation of Magnetic Skyrmions for Neuromorphic Computing Applications
}

This paper was downloaded from TechRxiv (https://www.techrxiv.org).

\section{LICENSE}

CC BY 4.0

SUBMISSION DATE / POSTED DATE

$31-10-2021 / 05-11-2021$

\section{CITATION}

Lone, Aijaz; Ganguly, Arnab; Divyanshu, Divynahsu; Amara, Selma; Das, Govind; Fariborzi, Hossein (2021): Creation and Annihilation of Magnetic Skyrmions for Neuromorphic Computing Applications. TechRxiv. Preprint. https://doi.org/10.36227/techrxiv.16910503.v1

$\mathrm{DOI}$ 


\title{
Creation and Annihilation of Magnetic Skyrmions for Neuromorphic Computing Applications
}

\author{
Aijaz H. Lone ${ }^{1}$, Arnab Ganguly ${ }^{2}$, Divyanshu ${ }^{1}$, Selma Amara ${ }^{1}{\text { Gobind } \text { Das }^{2} \text { and H. Fariborzi }}^{1}$ \\ ${ }^{1}$ Computer, Electrical and Mathematical Science and Engineering Division, King Abdullah University of Science and \\ Technology, Thuwal, Saudi Arabia. \\ ${ }^{2}$ Department of Physics, Khalifa University, Abu Dhabi 12788, United Arab Emirates.
}

In this work we present the creation, annihilation and dynamics of a topologically protected magnetic structure, a skyrmion, for neuromorphic computing application. We study the effect of Dzyaloshinskii Moriya interaction (DMI) and surface anisotropy on the skyrmion density. The relation between skyrmion annihilation threshold anisotropy $K_{\text {th }}$ and DMI coefficient is evaluated. Furthermore, the skyrmion diameter dependence on these two parameters is studied. Using MOKE analysis we study the effect of external magnetic field on the skyrmion density and predict the threshold magnetic field for the transition of magnetic texture from Labriynth domains to skyrmions. These results are further supported by the MuMax simulations. The spin orbit torque SOT manipulation of skyrmion size and density is also presented for skyrmion applications in the race-track memory and neuromorphic computing. Motivated by the results, we propose a Skyrmionic neuromorphic device and using SOT switching mechanism, show its applicability as spintronic synapse and neuron. The MuMax simulations are coupled to the Non- Equilibrium Green's Function formalism to model the neuron and synapse behavior. Finally, we conclude with the possibility of using these devices for pattern recognition and other unconventional computing paradigms.

Index Terms - Skyrmions, DMI, Magnetic tunnel junction (MTJ), MOKE, MuMax, NEGF, Neuromorphic mimetic devices and Unconventional Computing

\section{INTRODUCTION}

Magnetic Skyrmions are topologically protected swirling structures induced by chiral interactions in noncentrosymmetric magnetic compounds or thin films with broken inversion symmetry[1]. The Dzyaloshinskii Moriya Interaction (DMI), as the chiral antisymmetric exchange interaction responsible for formation of these textures, has roots in the strong spin orbit coupling at the heavy metal/ferromagnetic interface (HM/FM) with broken inversion symmetry[2]. These magnetic textures emerge out of the competition among different energy terms. The exchange and anisotropy terms prefer parallel alignment of spins whereas the DMI and dipolar energy terms prefers non-collinear alignment of the spins[3]. In asymmetric ferromagnetic multilayer system, the DMI is induced by the high interfacial spin orbit coupling resulting from symmetric breaking. Since DMI and anisotropy are material property and geometry dependent, so, in order to stabilize these skyrmions and define a particular chirality, combination of different HM/FM structures are being investigated[4]. The other important parameters and effects that define the size and density of skyrmions include Zeeman field and the exchange coefficient. The spintronic devices based on these textures promise increased density and energy efficient data storage due to small nanometric size and topological protection[5]. These textures can be driven by very low depinning current densities [6] and show scalability down to 1 $\mathrm{nm}[7]$. Owing to these properties the magnetic skyrmions are being highly explored for data storage and computing[8]. Particularly, the skyrmionics devices show a lot of potential for their applicability in unconventional computing such as Neuromorphic computing[9], reversible computing [10] etc. Neuromorphic computing is inspired by the performance and energy efficiency of the brain[11]. It involves the neuromimetic devices such as neurons, responsible for the computing. The synapses store the information in terms of weights. Among the different beyond CMOS devices, spintronics devices, especially magnetic tunnel junction MTJ, has found wide interests in the neuromorphic computing [12]. In recent years different neuromorphic computing systems with MTJ devices based on the skyrmions have been proposed such as skyrmion neurons [13] and skyrmion synapses [14].

In this work we first study the most important aspects of the skyrmionics that involves creation (nucleation), annihilation and motion of the skyrmions. We study the effect of DMI and surface anisotropy on the skyrmion density and evaluate the relationship between skyrmion annihilation threshold anisotropy $\mathrm{K}_{\mathrm{th}}$ and DMI coefficient. Furthermore, the skyrmion diameter dependence on these two parameters is studied. Using MOKE analysis we study the effect of external magnetic field on the skyrmion density and predict the threshold magnetic field for the transition of magnetic texture from Labriynth domains to skyrmions. These results are further supported by 
our MuMax simulations. Furthermore, we show the spin orbit torque SOT manipulation of skyrmion size and density in creation and deletion of the skyrmions, and at the same time, SOT drives these skyrmions. Thus, promising for its applications in the race-track memory and neuromorphic computing. Motivated by these results, we propose a Skyrmionic neuromorphic device and using SOT switching mechanism, we show its applicability as spintronic synapse and neuron. Finally, we conclude with the possibility of using these devices for pattern recognition and other unconventional computing paradigms.

The rest of the paper is presented as follows. The sample preparation and characterization are discussed in section II. In section III we explore the device modeling using micromagnetics and Non-Equilibrium Green's Function formalism. In section IV we present our results and discussion on skyrmion size and density dependence on different energy terms. Section V introduces our neuro-mimetic devices (skyrmion synapse and neuron) and their electrical manipulation for system level applications. The last section includes concluding remarks and a discussion on future prospects of our work.

\section{FABRICATION AND CHARACTERIZATION}

Thin-film multilayer of Ta $(5 \mathrm{~nm}) / \mathrm{IrMn}(5 \mathrm{~nm}) / \mathrm{CoFeB}(1.04$ $\mathrm{nm}) / \mathrm{MgO}(2 \mathrm{~nm}) / \mathrm{Ta} 2(\mathrm{~nm})$ is deposited on thermally oxidized Si substrates using Singulus DC/RF magnetron sputtering. Here the $\mathrm{CoFeB}$ thickness is a curtail parameter providing suitable anisotropy for generating high-density skyrmions. The sputtering conditions are carefully optimized for obtaining PMA. The sample is treated with a post-deposition annealing at $250^{\circ} \mathrm{C}$ for $30 \mathrm{~min}$ for a further enhancement of the PMA. The experiment is performed using magneto-optical Kerr effect (MOKE) microscopy in polar geometry. A differential Kerr imaging is performed to observe the magnetic domains and to eliminate the contribution of any nonmagnetic intensities. Square pulses of the magnetic field are simultaneously applied both in-plane and out-of-plane of the sample using two independent electromagnets. The sample exhibits labyrinth domain structure in absence of any magnetic field. The magnetization is first saturated in one perpendicular direction by a sufficiently large out-of-plane field. A reference p-MOKE image is captured in this state. In the next step, $H_{z}$ is reduced to the desired value accompanied by an in-plane field $\left(H_{x}\right)$ as required. A second p-MOKE image is captured in this state. The magnetic image of the final state is obtained by the differential image with respect to its reference image.

\section{DEVICE MODELING}

The magnetic skyrmions are defined by their topological number or skyrmion number $\mathrm{Q}$, calculated by [15]:

$$
Q=\frac{1}{4 \pi} \iint \boldsymbol{m} \cdot\left(\frac{\partial \boldsymbol{m}}{\partial x} \times \frac{\partial \boldsymbol{m}}{\partial y}\right) d x d y
$$

The spins are projected on the $\mathrm{x}-\mathrm{y}$ plane and normalized magnetization vector $\boldsymbol{m}$ can be determined by the radial function $\theta$, Vorticity $Q_{v}$ and helicity $Q_{h}$

$m(r)=\left[\sin (\theta) \cos \left(Q_{v} \varphi+Q_{h}\right), \sin (\theta) \sin \left(Q_{v} \varphi+\right.\right.$

$$
\left.\left.Q_{h}\right), \cos (\theta)\right]
$$

Where, helicity number is related to skyrmion number by [8].

$$
Q=\frac{Q_{v}}{2}\left[\lim _{r \rightarrow \infty} \cos (\theta(r))-\cos (\theta(0))\right]
$$

The micromagnetic simulations were carried out using MuMax having Landau-Lipshitz-Gilbert (LLG) equation as the basic magnetization dynamics computing unit [16]. The LLG describes the magnetization evolution by

$$
\frac{d \widehat{\boldsymbol{m}}}{d t}=\frac{-\gamma}{1+\alpha^{2}}\left[\boldsymbol{m} \times \boldsymbol{H}_{\text {eff }}+\boldsymbol{m} \times\left(\boldsymbol{m} \times \boldsymbol{H}_{\text {eff }}\right)\right]
$$

Where, $\boldsymbol{m}$ is the normalized magnetization vector, $\gamma$ is the gyromagnetic ratio, $\alpha$ is the Gilbert damping coefficient and

$$
\boldsymbol{H}_{\text {eff }}=\frac{-1}{\mu_{0} M_{S}} \frac{\delta \boldsymbol{E}}{\delta \boldsymbol{m}}
$$

is the effective magnetic field around which magnetization process. The total magnetic energy of the free layer includes Exchange energy, Zeeman energy, Uniaxial anisotropy energy, demagnetization energy and DMI energy [17][18].

$$
E(\boldsymbol{m})=\int_{V}\left[A(\nabla \boldsymbol{m})^{2}-\mu_{0} \boldsymbol{m} \cdot H_{e x t}-\frac{\mu_{0}}{2} \boldsymbol{m} \cdot H_{d}-K_{u}(\widehat{u} \cdot \boldsymbol{m})+\right.
$$

Where, $\mathrm{A}$ is the exchange stiffness, $\mu_{0}$ is the permeability, $\mathrm{Ku}$ is the anisotropy energy density, $H_{d}$ the demagnetization field $H_{\text {ext }}$ is the external field and the DMI energy density is computed by

$$
\varepsilon_{D M}=D\left[m_{z}(\nabla \cdot \boldsymbol{m})-(\boldsymbol{m} \cdot \nabla) \cdot \boldsymbol{m}\right]
$$

we add spin orbit torque as a custom field term in MuMax [18].

$$
\begin{gathered}
\boldsymbol{\tau}_{\text {SOT }}=-\frac{\gamma}{1+\alpha^{2}} a_{J}[(1+\xi \alpha) \boldsymbol{m} \times(\boldsymbol{m} \times \boldsymbol{p})+(\xi-\alpha)(\boldsymbol{m} \times \boldsymbol{p})] \\
a_{J}=\left|\frac{\hbar}{2 M_{S} e \mu_{0}} \frac{\theta_{S H}}{d}\right| \quad \text { and } \boldsymbol{p}=\operatorname{sign}\left(\theta_{S H}\right) \boldsymbol{j} \times \boldsymbol{n}
\end{gathered}
$$


Where $\theta_{S H}$ is the spin Hall coefficient of the material, $j$ is the current density and $\mathrm{d}$ is the free layer thickness.

The synapse resistance and neuron output voltage are computed using Non-Equilibrium Green's Function formalism. We take the magnetization profile of the free layer and feed it to our NEGF model which computes the resistance of the MTJ device as follows[19][20].

$$
R_{s y n}=\frac{V_{s y n}}{I_{s y n}}
$$

Where,

$$
I_{s y n}=\operatorname{trace}\left\{\sum_{k_{t}} C_{\sigma} \frac{i}{\hbar}\left\{\begin{array}{l}
H_{k^{\prime} k+1} G^{n}{ }_{k+1, k} \\
-G^{n}{ }_{k, k+1} H_{k+1, k}
\end{array}\right\}\right\}
$$

The $H_{k}$ defines the kth lattice site in device Hamiltonian, $G^{n}{ }_{k}$ is the electron correlation at kth site which gives the electron density.

\section{RESULTS AND DISCUSSION}

The skyrmion size and density dependence on DMI and anisotropy is shown in Fig. 1. We observe that the skyrmion density increases with the magnitude of DMI coefficient as shown in Fig. 1(a). Indicating the crucial role of DMI crucial in skyrmion stabilization. However, with increasing anisotropy the skyrmion density is reduced and skyrmions start annihilating at their respective threshold anisotropy $K_{\text {th }}$. Because the DMI wants spins to align in non-collinear fashion but anisotropy field prefers the collinear alignment. Thus, depending upon the magnitude of the DMI, the skyrmion annihilation threshold anisotropy also varies. As shown in Fig. 1(b) the micromagnetic results are in agreement with the critical DMI $D_{C}$ for the skyrmion equation expressed as [21].

$$
D_{c}=4 \sqrt{A\left(K_{u}+\left(H_{Z}-0.5 N_{Z} \mu_{0} M_{S}\right) M_{S}\right)} / \pi
$$

In order to fit our results, we write the equation as

$$
D_{c^{\prime}}=4 \sqrt{A\left(K_{u}+\left(H_{Z}-k \mu_{0} M_{S}\right) M_{S}\right)} / \pi
$$

Where, $k$ is the fitting variable, signifying the role of demagnetization field on the stabilization of skyrmions. We observe that the MuMax and analytical results exactly match each other if we consider the demagnetization field impact on skyrmion stability to be increasing with coefficient of DMI. As shown in the Fig. 1(b) we use the $\mathrm{k}$ as a fitting parameter between Micromagnetics results and the analytical equation for the critical DMI for skyrmion formation. The optimal fit obtained for $\mathrm{k}=0.65$ till $\mathrm{Ku}$ is $9 \mathrm{MJm}^{-3}$ and $\mathrm{k}=0.83$ for $\mathrm{Ku}>$ $9 \mathrm{MJm}^{-3}$ clearly, depicting the role of demagnetization field in skyrmion stabilization for higher anisotropies. Furthermore, as shown in Fig. 2 we can clearly observe that the diameter of the skyrmion reduces with increasing the anisotropy as anisotropy wants spins to be aligned collinearly in $\mathrm{z}$ direction. Figure 3(a) shows magnetic texture at different $H_{Z}$. The field value $\mathrm{H}_{\mathrm{Z}}$ is mentioned in the top right corner of each figure. In addition to $\mathrm{H}_{\mathrm{z}}$ a constant in-plane magnetic field $H_{x}=20 \mathrm{Oe}$ is also applied.

(a)

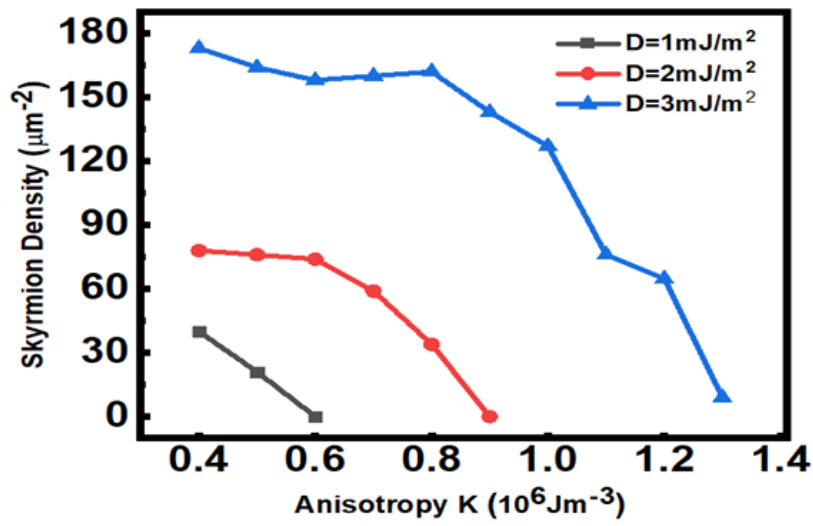

(b)

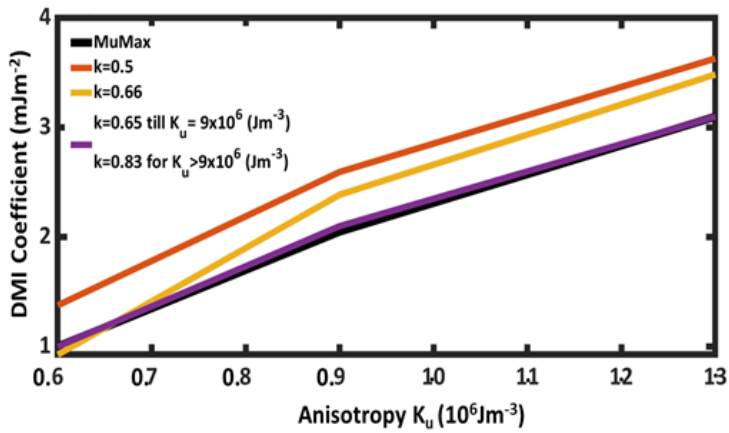

Fig. 1(a). Skyrmion density as a function of anisotropy and DMI coefficient. (b) Critical DMI coefficient versus the anisotropy. The MuMax results are matched to analytical expression [21] after fitting. Here, $\mathrm{k}$ is the fitting parameter.

(a)

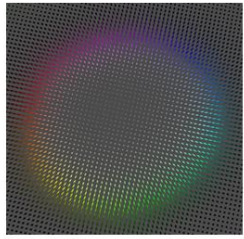

$\mathrm{KS} 1=0.5 \times 10^{6}\left(\mathrm{Jm}^{-3}\right)$

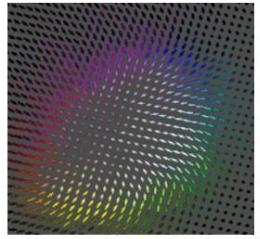

$\mathrm{KS} 2=0.6 \times 10^{6}\left(\mathrm{Jm}^{-3}\right)$

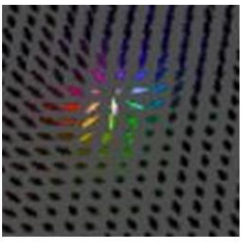

$\mathrm{KS} 3=0.7 \times 10^{6}\left(\mathrm{Jm}^{-3}\right)$

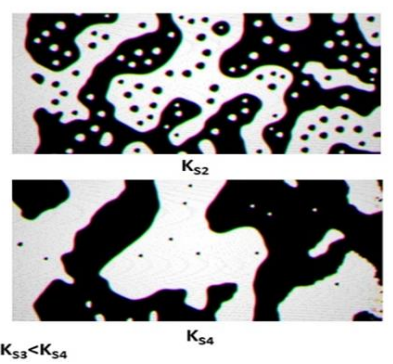

Fig. 2(a). Skyrmion diameter reducing with increasing anisotropy. (b) Magnetization texture of free layer depicting that density of skyrmion decreases with increasing anisotropy.

The white and black contrast correspond to $\uparrow$ and $\downarrow$ domains respectively. We observe that the long labyrinth domains are 
broken into smaller structures by increasing $H_{z}$ from 5 Oe to 12 Oe. At $H_{z}=20$ Oe, it is further divided into tiny particle structures called skyrmions. A further increase in the field reduces skyrmion density $\left(H_{z}=24 \mathrm{Oe}\right)$ followed by a complete annihilation of skyrmions. In our experiment, we systematically study the density of skyrmions as a function of $H_{x}$ and $H_{z}$. The results are summarized in Figure 3(b). Here we see that the density of skyrmions maximizes around $20 \mathrm{kOe}$ which is almost independent of the $H_{x}$. The skyrmion density increases with increasing $H_{x}$ and a broader window of $H_{z}$ is obtained for the creation of skyrmions. These results are further supported by our MuMax simulations as shown in Fig. 3(b). Note, in simulations we observe zero field skyrmion density and its rolling behavior with increasing field. The results in Fig. (1-2) provide a window into the optimization of material parameters and stack geometry in order to tweak the skyrmion density and diameter for its application in memory and logic.

(a)
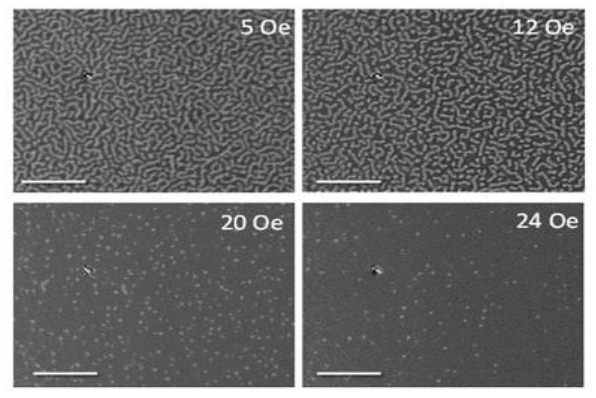

$4 \mathrm{Oe}$

(b)

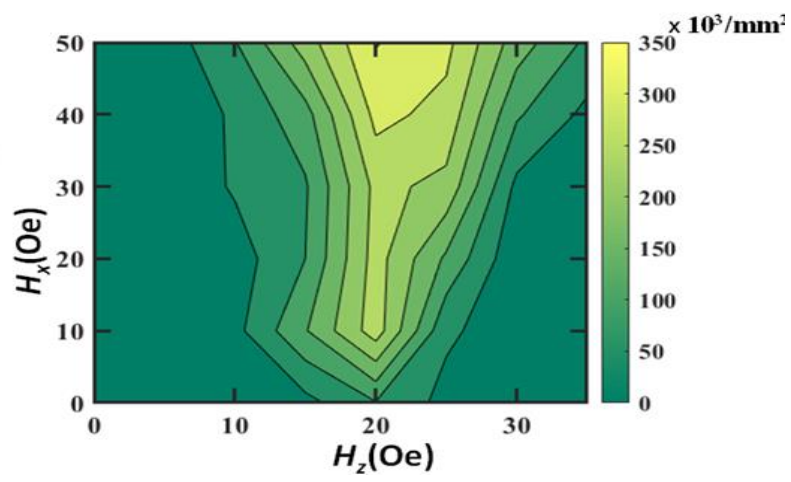

(c)

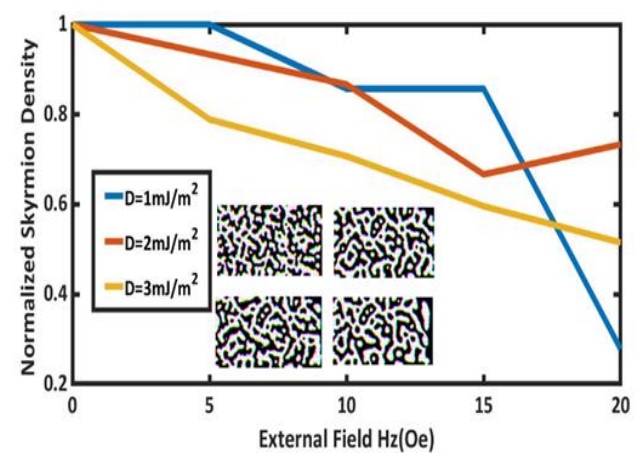

Fig. 3(a) MOKE images showing the skyrmion density dependence on the external field. (c) MuMax simulations in support of the MOKE results.

\section{NEUROMIMETIC SKYRMION SYNAPSE AND NEURON}

Advancing our discussion based on DMI, anisotropy and Zeeman field control of skyrmion size and density. In Fig. 4(a) we propose an MTJ neuromorphic showing synaptic and Leaky integrate and fire (LIF) neuron behavior. The free layer (FL) and oxide layer (TB) of the MTJ are extended as compared to the fixed layer (PL). The heavy metal HM/free layer FL/ and oxide layer TB combination provide the required asymmetry for DMI and SOT. The SOT generated at the HM/FL interface shows a dual effect on the skyrmion density and its motion. The SOT field has a similar effect on skyrmion density as Zeeman field. The SOT manipulation of skyrmion size and density is shown in Fig. 2 (b), were increasing the current via the heavy metal initially increases the skyrmion density from $21 \mu \mathrm{m}^{-2}$ (at $2 \times 10^{11} \mathrm{~A} / \mathrm{m}^{2}$ ) to $40 \mu \mathrm{m}^{-2}$ (at $8 \times 10^{11} \mathrm{~A} / \mathrm{m}^{2}$ ). Increasing charge current further results in decrease of the skyrmion density. SOT also results in skyrmion motion across the free layer. Thus, we show that SOT can help in nucleation and deletion of the skyrmions, and at the same time, drive the skyrmions for its applications in the race-track memory and or neuromorphic computing. Furthermore, to show the synaptic behavior mimicked by this device we use MuMax Coupled NEGF simulations.

(a)

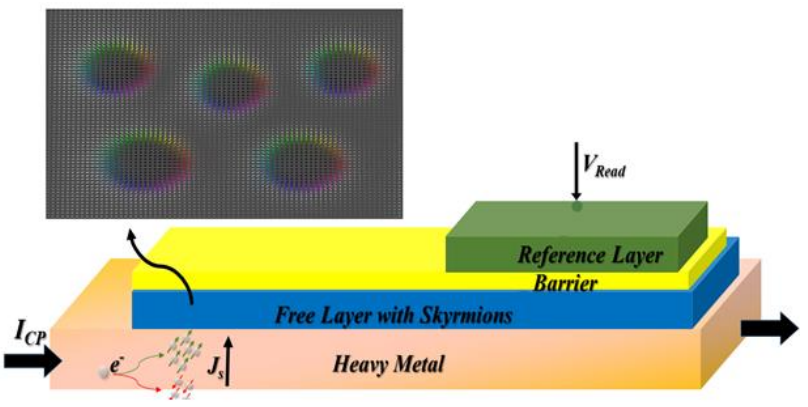

(b)

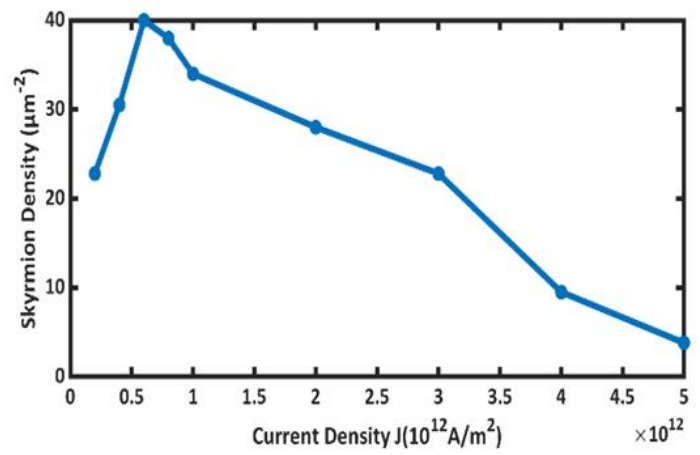

Fig. 4(a) Skyrmion based MTJ-synapse. (b) SOT control of the Skyrmion density.

We present the Skyrmion synapse with alternate positive and negative current pulses via heavy metal. As shown in Fig. 5(a) the resistance (synaptic weight) of the device in the beginning 
varies gradually till $5 \mathrm{~ns}$. During this time skyrmions are nucleated and stabilized in presence of low amplitude current pulses $2 \times 10^{11} \mathrm{~A} / \mathrm{m}^{2}$ (current density). After $5 \mathrm{~ns}$ we apply

$8 \times 10^{11} \mathrm{~A} / \mathrm{m}^{2}$ positive current pulse followed a negative current pulse of same magnitude. The positive current increases the synaptic resistance from 460 to 650 almost linearly. Thus, causing the synaptic depression conductance (weight) is decreased. Similarly, a negative current pulse decreases resistance thus, helps in the synaptic potentiation of the skyrmion device. The synaptic potentiation/depression plays a crucial role in the learning of the neural network. So, these devices show a good potential in realization of system level neuromorphic computing.

Furthermore, we modify the device structure for its applications as the neuron. We create a constriction in the free layer above which oxide and fixed layer are placed. As shown in the magnetic texture of the free layer in Fig. 5(c), before the beginning of constriction the current has only $\mathrm{x}$ component but at the constriction the current density splits into $\mathrm{x}$ and $\mathrm{y}$ component. Usually, in the independent ferromagnetic film the skyrmions deviate their path as they move in $\mathrm{x}$ direction. But, due to a current component in the transverse (y) direction, the skyrmions try to align back to the straight path as shown in Fig. 5(c). When presented with a current pulse the skyrmions move forward and are detected by the MTJ. In absence of the current, skyrmions show a small leaky behavior and move backwards. Fig. 5(b) shows this leaky-integrate and fire behavior of the skyrmion device. As the number of pulses increases the neuron output voltage increases and depending upon the threshold value the neuron fires by generating a spike. In this device we for illustrative purpose keep the threshold at around $57.5 \mathrm{mV}$.

(a)

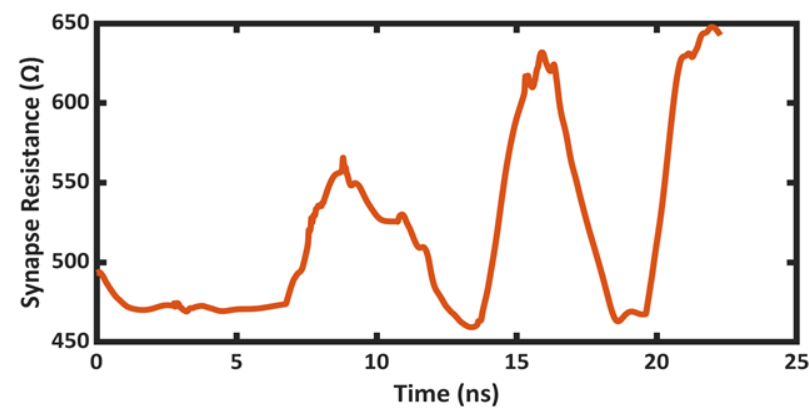

(b)

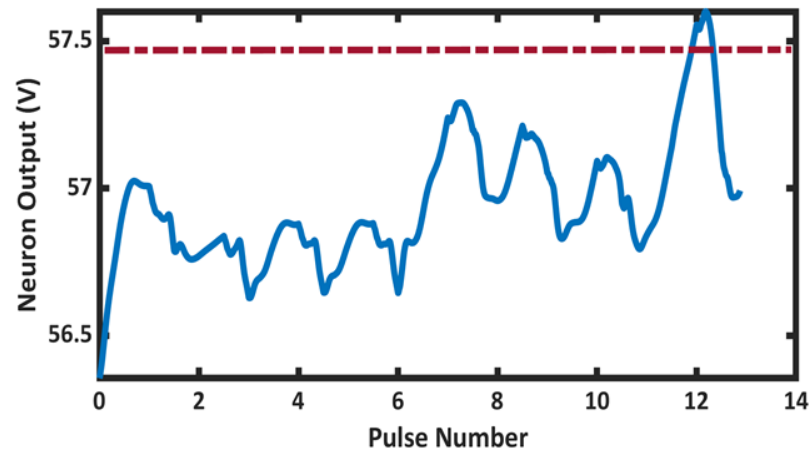

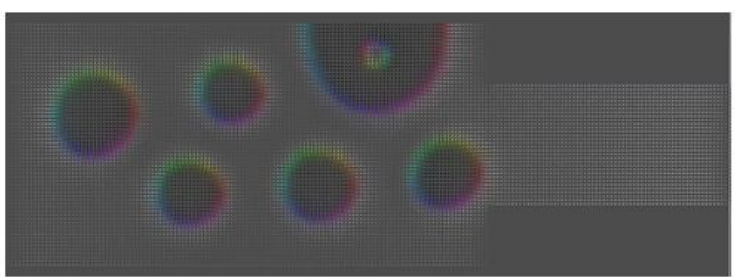

$\mathrm{t}=2 \mathrm{~ns}$

(c)

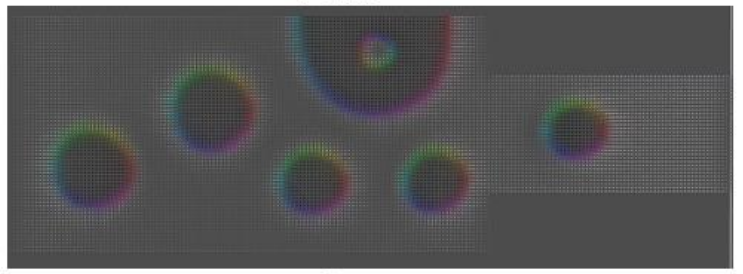

$\mathrm{t}=8 \mathrm{~ns}$

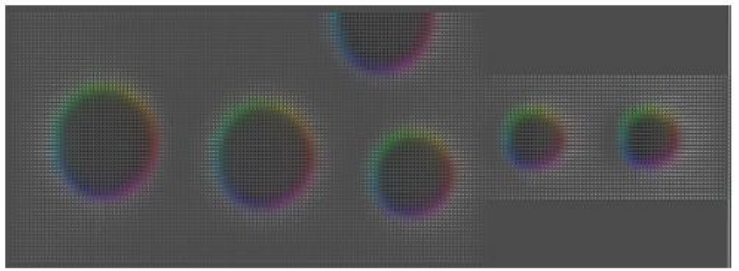

$\mathrm{t}=12 \mathrm{~ns}$

Fig. 5(a) Skyrmion based MTJ synapse showing potentiation and depression in presence of positive and negative current pulses. (b) Skyrmion based MTJ neuron showing leaky integrate and fire LIF neuron behavior. (c) Neuron free layer magnetic texture. The constriction helps skyrmions to move in the right direction and get detected by fixed layer.

\section{CONCLUSION}

In this paper the micromagnetic and experimental study on the nucleation, annihilation and motion of the skyrmion is presented. We evaluated the relation between DMI and anisotropy in the process of skyrmion nucleation and skyrmion diameter. External magnetic field plays a crucial role in the skyrmion stabilization as already found in the literature. We perform both experimental characterization and micromagnetic simulations to figure out the critical field values for skyrmion nucleation. Also, the existence of zero field skyrmion density is shown. These results are important for realization of skyrmion memory and logic devices. Furthermore, we show the SOT control of nucleation, annihilation and motion of the skyrmions. Based on the SOT control of the skyrmions, we propose neuromorphic skyrmionic devices such as skyrmion synapse and neuron. The synaptic potentiation and depression in these devices is also presented. The leaky integrate and fire LIF neuron realization is also shown. Thus, these results and the devices show promise to energy efficient memory, logic and unconventional computing paradigms. Presently we are working on the neuromorphic circuit realization based on the proposed devices.

\section{ACKNOWLEDGEMENT}

[1] A. Fert, N. Reyren, and V. Cros, "Magnetic skyrmions: Advances in physics and potential applications," Nat. Rev. Mater., vol. 2, 2017, 
doi: $10.1038 /$ natrevmats.2017.31.

[2] S. Woo et al., "Deterministic creation and deletion of a single magnetic skyrmion observed by direct time-resolved X-ray microscopy," Nat. Electron., vol. 1, no. 5, pp. 288-296, 2018, doi: 10.1038/s41928-018-0070-8.

[3] A. Bernand-Mantel, C. B. Muratov, and T. M. Simon, "Unraveling the role of dipolar versus Dzyaloshinskii-Moriya interactions in stabilizing compact magnetic skyrmions," Phys. Rev. B, vol. 101, no. 4, p. 45416, 2020, doi: 10.1103/PhysRevB.101.045416.

[4] M. Ma et al., " Enhancement of zero-field skyrmion density in $[\mathrm{Pt} / \mathrm{Co} / \mathrm{Fe} / \mathrm{Ir}] 2$ multilayers at room temperature by the first-order reversal curve ," J. Appl. Phys., vol. 127, no. 22, p. 223901, 2020, doi: $10.1063 / 5.0004432$.

[5] S. Luo and L. You, "Skyrmion devices for memory and logic applications," APL Mater., vol. 9, no. 5, pp. 1-11, 2021, doi: $10.1063 / 5.0042917$.

[6] J. Zang, M. Mostovoy, J. H. Han, and N. Nagaosa, "Dynamics of Skyrmion crystals in metallic thin films," Phys. Rev. Lett., vol. 107, no. 13, pp. 1-5, 2011, doi: 10.1103/PhysRevLett.107.136804.

[7] X. S. Wang, H. Y. Yuan, and X. R. Wang, "A theory on skyrmion size," Commun. Phys., vol. 1, no. 1, pp. 1-7, 2018, doi: 10.1038/s42005-018-0029-0.

[8] W. Kang, Y. Huang, X. Zhang, Y. Zhou, and W. Zhao, "SkyrmionElectronics: An Overview and Outlook," Proc. IEEE, vol. 104, no. 10, pp. 2040-2061, 2016, doi: 10.1109/JPROC.2016.2591578.

[9] K. M. Song et al., "Skyrmion-based artificial synapses for neuromorphic computing," Nat. Electron., vol. 3, no. 3, pp. 148-155, 2020, doi: 10.1038/s41928-020-0385-0.

[10] M. Chauwin et al., "Skyrmion Logic System for Large-Scale Reversible Computation,” Phys. Rev. Appl., vol. 12, no. 6, pp. 1-24, 2019, doi: 10.1103/PhysRevApplied.12.064053.

[11] S. Li et al., "Emerging neuromorphic computing paradigms exploring magnetic skyrmions," Proc. IEEE Comput. Soc. Annu. Symp. VLSI, ISVLSI, vol. 2018-July, pp. 539-544, 2018, doi: 10.1109/ISVLSI.2018.00104.

[12] G. Srinivasan, A. Sengupta, and K. Roy, "Magnetic Tunnel Junction Based Long-Term Short-Term Stochastic Synapse for a Spiking Neural Network with On-Chip STDP Learning," Sci. Rep., vol. 6, no. June, pp. 1-13, 2016, doi: 10.1038/srep29545.

[13] J. Deng, V. P. K. Miriyala, Z. Zhu, X. Fong, and G. Liang, "VoltageControlled Spintronic Stochastic Neuron for Restricted Boltzmann Machine with Weight Sparsity," IEEE Electron Device Lett., vol. 41, no. 7, pp. 1102-1105, 2020, doi: 10.1109/LED.2020.2995874.

[14] A. F. Vincent et al., "Spin-transfer torque magnetic memory as a stochastic memristive synapse for neuromorphic systems," IEEE Trans. Biomed. Circuits Syst., vol. 9, no. 2, pp. 166-174, 2015, doi: 10.1109/TBCAS.2015.2414423.

[15] K. Everschor-Sitte, J. Masell, R. M. Reeve, and M. Kläui, "Perspective: Magnetic skyrmions - Overview of recent progress in an active research field," J. Appl. Phys., vol. 124, no. 24, 2018, doi: 10.1063/1.5048972.

[16] J. Leliaert, M. Dvornik, J. Mulkers, J. De Clercq, M. V. Milošević, and B. Van Waeyenberge, "Fast micromagnetic simulations on GPU - Recent advances made with mumax3," J. Phys. D. Appl. Phys., vol. 51, no. 12, 2018, doi: 10.1088/1361-6463/aaab1c.

[17] A. Vansteenkiste, J. Leliaert, M. Dvornik, M. Helsen, F. GarciaSanchez, and B. Van Waeyenberge, "The design and verification of MuMax3," AIP Adv., vol. 4, no. 10, 2014, doi: 10.1063/1.4899186.

[18] F. Büttner et al., "Field-free deterministic ultrafast creation of magnetic skyrmions by spin-orbit torques," Nat. Nanotechnol., vol. 12, no. 11, pp. 1040-1044, 2017, doi: 10.1038/nnano.2017.178.

[19] A. H. Lone, S. Shringi, K. Mishra, and S. Srinivasan, "CrossSectional Area Dependence of Tunnel Magnetoresistance, Thermal Stability, and Critical Current Density in MTJ," IEEE Trans. Magn., vol. 57, no. 2, 2021, doi: 10.1109/TMAG.2020.3039682.

[20] S. Datta, Quantum Transport: Atom to Transistor, Cambridge University Press. 2005.

[21] S. Rohart and A. Thiaville, "Skyrmion confinement in ultrathin film nanostructures in the presence of Dzyaloshinskii-Moriya interaction," Phys. Rev. B - Condens. Matter Mater. Phys., vol. 88, no. 18, pp. 1-8, 2013, doi: 10.1103/PhysRevB.88.184422. 